\title{
Excitability Mediated by Localized Structures in Kerr Cavities
}

\author{
Damià Gomila, Adrian Jacobo, Manuel A. Matías, and Pere Colet \\ Instituto Mediterraneo de Estudios Avanzados, IMEDEA (CSIC-UIB) \\ Campus Universitat Illes Balears, 07122 Palma de Mallorca, Spain
}

\begin{abstract}
We characterize a scenario where localized structures in nonlinear optical cavities display an oscillatory behavior which becomes unstable leading to an excitable regime. Excitability emerges from spatial dependence since the system locally is not excitable. We show the existence of different mechanisms leading to excitability depending on the profile of the pump field.
\end{abstract}

DOI: 10.2529/PIERS060907134206

Localized structures (LS) in dissipative optical cavities arise as a consequence of the interplay between diffraction, nonlinearity, driving, and dissipation [1]. These structures, also known as cavity solitons, are unique once the parameters of the system have been fixed. This fact makes this structures potentially useful in optical storage and processing of information [2,3]. LS may develop a number of instabilities, for instance their amplitude can oscillate in time while remaining static in space. Here we report on a novel regime of excitability associated to the existence of localized structures in a nonlinear optical system [4,5]. Excitability has been found in a variety of systems [6], including optical systems [7], and is characterized by a nonlinear response under applied external perturbation. Perturbations exceeding a certain threshold are able to elicit in the system a full and well defined response. Furthermore after one perturbation the system cannot be excited again within a refractory period of time. Excitability is behind excitation waves in heart tissue and the existence of action potentials in neurons, and, so, may confer new computational capabilities to optical systems beyond information storage.

In this paper we show the existence of different mechanisms leading to excitability depending on the profile of the pump field. For a homogeneous pump the mechanism leading to excitable behavior is a saddle-loop bifurcation through which an stable oscillating LS collides with an unstable LS [4]. For a system pumped by a localized Gaussian beam on top of homogeneous background the scenario is richer and one finds two different mechanisms leading to excitability. One is based on a saddleloop bifurcation as above while the other takes place through a saddle-node in the invariant circle (SNIC) bifurcation. This second mechanism has excitability threshold which can be much lower.

We consider a ring cavity filled with a nonlinear self-focusing Kerr medium pumped by an external field. In the mean field approximation, the dynamics of the electric field inside the cavity can be described by a single partial differential equation for the scaled slowly varying amplitude $E(\vec{x}, t)[8]$

$$
\partial_{t} E=-(1+i \theta) E+i \nabla^{2} E+E_{0}+i|E|^{2} E,
$$

where $E_{0}$ is the homogeneous driving field and $\theta$ is the cavity detuning. The homogeneous steady state solution of Eq. (1) is implicitly given by $E_{s}=E_{0} /\left(1+i\left(\theta-I_{s}\right)\right)$, where $I_{s}=\left|E_{s}\right|^{2}$. We use the intra-cavity background intensity $I_{s}$ together with $\theta$ as convenient control parameters. The homogeneous solution has a modulational instability (MI) at $I_{s}=1.0$ leading to the formation of subcritical hexagonal patterns.

The existence and dynamical properties of localized structures in this system, the so called Kerr cavity solitons (KCS), have been studied in [9] and references therein. Fig. 1 shows the region of existence of KCS in the $I_{s}-\theta$ parameter space. The KCS branch starts subcritically at the MI point. The middle-branch KCS is unstable and it has only a single unstable mode. There is a turning point (saddle-node) where an stable upper-branch KCS starts. The bifurcation diagram as function of $I_{s}$ for fixed $\theta$ is shown in Fig. 1. A similar diagram can be obtained for fixed $I_{s}$ as function of $\theta$. Increasing $I_{s}$ or $\theta$ the upper-branch KCS undergoes a Hopf bifurcation resulting in a periodically oscillating localized structure. The oscillation is such that it approaches the stable manifold of the middle-branch KCS and then escapes along the unstable manifold. As the detuning is increased the limit cycle goes closer and closer to the middle-branch KCS as illustrated in Fig. 2. At a critical value $\theta_{c}$ a global bifurcation takes place: the cycle touches the middle-branch KCS and 

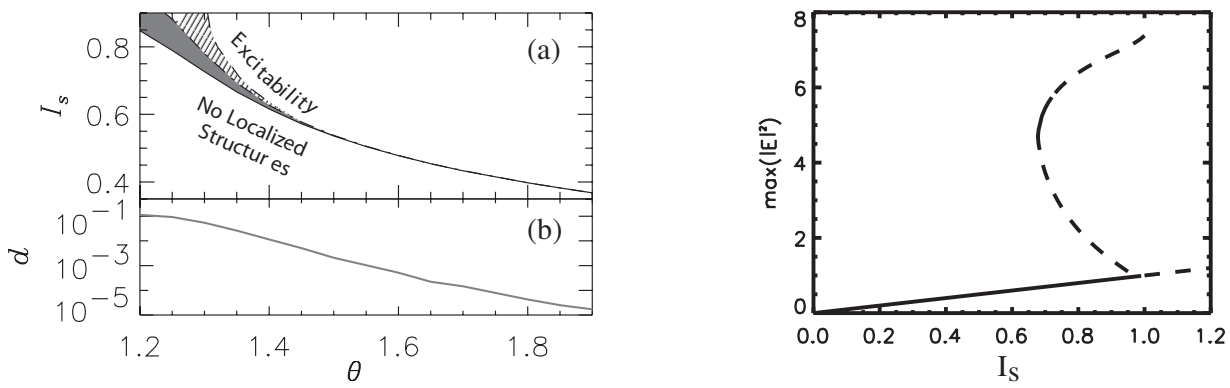

Figure 1: Left: (a) Phase diagram: $I_{s}$ vs. $\theta$ showing the different regimes. LS are stable in the shaded region and oscillate in the dashed one. The solid line indicates the saddle-node bifurcation where the LS are created, the dot-dashed the Hopf, and the dashed the saddle-loop where the oscillation is destroyed. (b) Distance between the saddle-node and Hopf lines. Right: Bifurcation diagram of KCS for $\theta=1.34$. The upper (middle) branch corresponds to stable (unstable) KCS. These branches are originated at a saddle-node bifurcation. The upper branch becomes Hopf unstable for larger values of $I_{s}$.
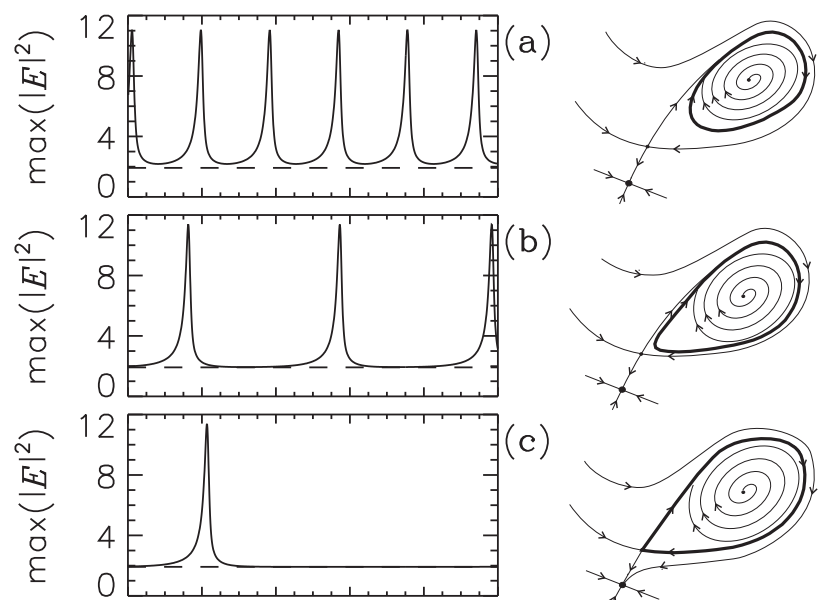

(c)
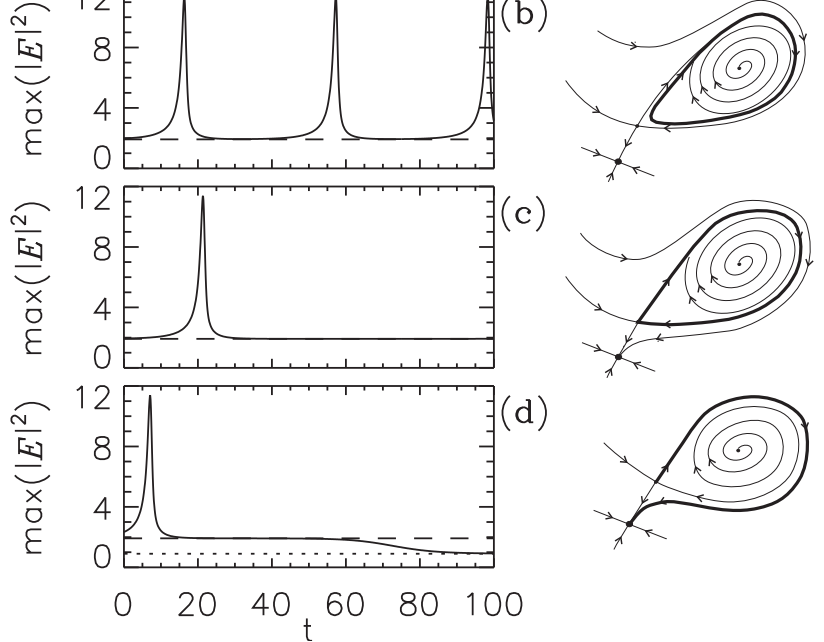

Figure 2: Left: LS maximum intensity as a function of time for increasing values of the detuning parameter $\theta$. From top to bottom $\theta=1.3,1.3047,1.30478592,1.304788 . I_{s}=0.9$. Right: Sketch of the phase space for each parameter value. The thick line shows the trajectory of the LS in phase space.

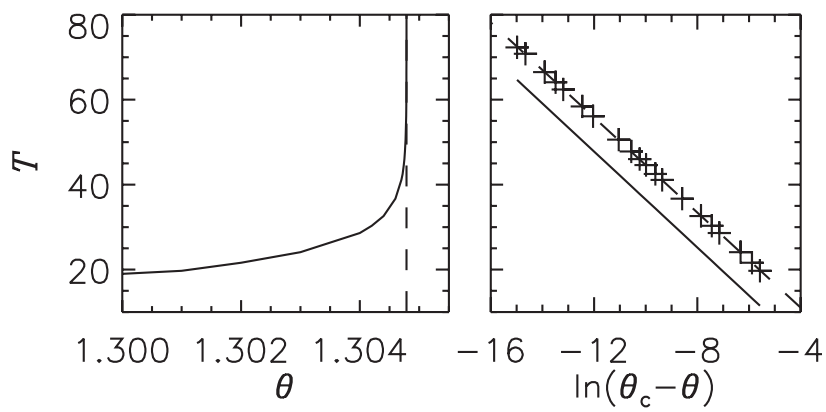

Figure 3: (a) Period of the limit cycle $T$ as a function of the detuning $\theta$ for $I_{s}=0.9$. The vertical dashed line indicate the threshold of the saddle-loop bifurcation $\theta_{c}=1.30478592$. (b) Period $T$ as a function of $\ln \left(\theta_{c}-\theta\right)$. Crosses correspond to numerical simulations while the solid line has a slope $1 / \lambda_{1}$ with $\lambda_{1}=0.177$ obtained from the linear stability analysis of the middle-branch LS. 


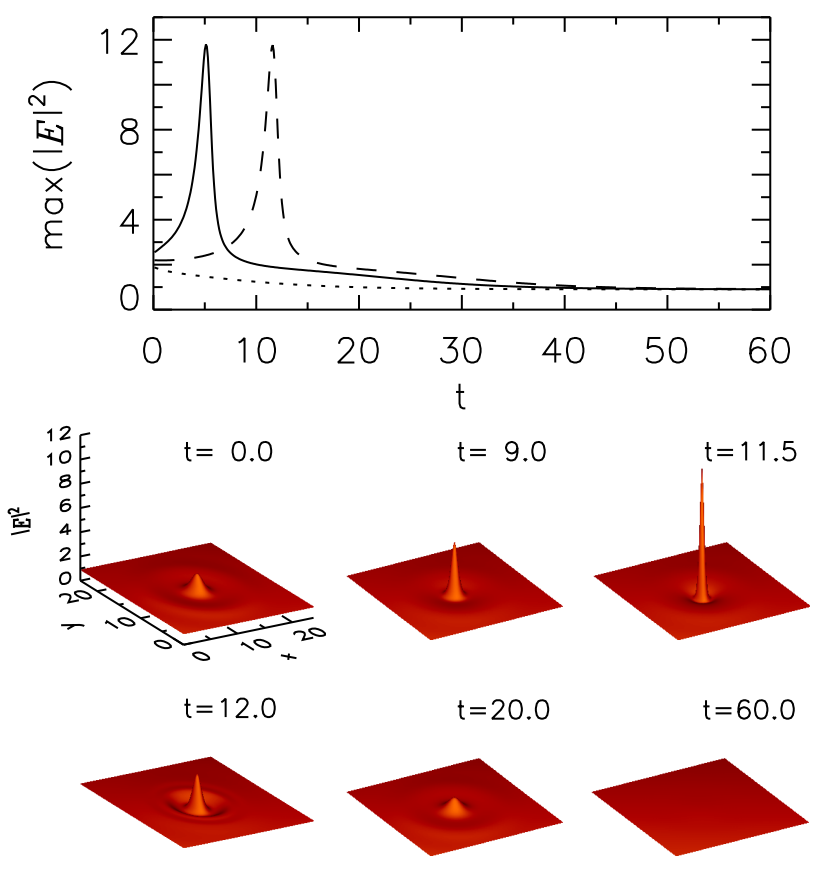

Figure 4: Evolution of a sub-threshold (dotted line) and two above-threshold perturbations, one close to the threshold (solid line) and one well above (dashed line). Top panel shows the time evolution of the maximum intensity, while the 3D plots show the transverse profile at different times for the dashed trajectory.

becomes a homoclinic orbit [Fig. 2(c)]. This is an infinite-period bifurcation called saddle-loop or homoclinic bifurcation. The saddle-loop has a characteristic scaling law that govern the period $T$ of the limit cycle as the bifurcation is approached: $T \sim-\ln \left(\theta_{c}-\theta\right) / \lambda$, where $\lambda$ is the unstable eigenvalue of the middle-branch KCS. We show that this scaling is verified in our system (Fig. 3).

Beyond the saddle-loop bifurcation the phase space shows a typical configuration presenting excitability [Fig. 2(d)]: it has a globally attracting fixed point (homogeneous solution), but localized disturbances (above the middle-branch KCS) can send the system on a long excursion in the phase space before returning to the fixed point (Fig. 4). The peak grows to a large value until the losses dominate the dynamics and stop the growth. Then decays exponentially until it disappears. A remnant wave is emitted out of the center dissipating the remaining energy. The long excursion in phase space is reminiscent of the coherence collapse phenomenon that arises in the $2 \mathrm{D}$ nonlinear Schrödinger equation. The Hopf instability of KCS in this limit has been studied in [10]

All this scenario is organized by a co-dimension two Takens-Bogdanov bifurcation point. In the limit of large detuning, the saddle-node, Hopf and saddle-loop bifurcation lines meet asymptotically, at $I_{s}=0$ as shown in Fig. 1. It is known that the intersection of a saddle-node line with a Hopf line is a Takens-Bogdanov (TB) codimension-2 bifurcation point [11]. The unfolding around a TB point leads to a saddle-loop bifurcation line [11]. So, this unfolding fully explains the observed scenario, where our formally infinite-dimensional system appears to be perfectly described by a dynamical system in the plane.

We should emphasize that, in absence of spatial degrees of freedom, the system described by Eq. (1) is not an excitable system. Excitability arises here as an emergent property linked to the spatial degrees of freedom in the system. The mechanism for excitability is based on the dynamics of the localized structure by itself.

We consider in the following the case in which the system is pumped by a field $E_{I}$ consisting of a narrow Gaussian beam on top of homogeneous background $E_{0}: E_{I}=E_{0}+H \exp \left(-r^{2} / r_{0}^{2}\right)$ where $H=\sqrt{\left(I_{s}+I_{s h}\right)\left[1+\left(\theta-I_{s}-I_{s h}\right)^{2}\right]}-E_{0}$. The control parameters are $I_{s}$ associated to the background intensity, the detuning $\theta$, and the new $I_{s h}$ associated to Gaussian beam. Now the translational symmetry of the system (and also of its solutions) is broken, and the fundamental solution, no longer homogeneous, exhibits a small bump (small when compared to the truly localized structures), which is the system response to the Gaussian perturbation. The bifurcation diagram for fixed $I_{s h}$ and $\theta$ is shown in Fig. 5 .

The maximum of the fundamental solution increases as we increase $I_{s}$ until a saddle-node bi- 


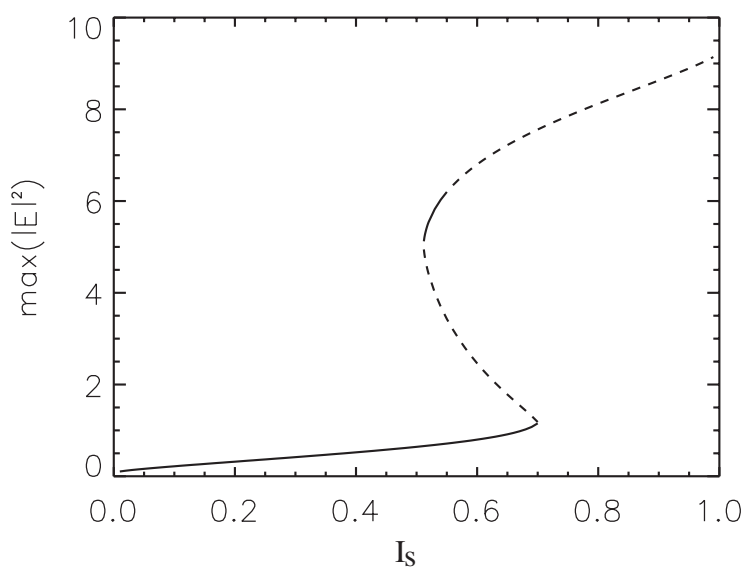

Figure 5: Bifurcation diagram $\max \left(|E|^{2}\right)$ vs $I_{s}$ for pump consisting of a Gaussian beam on top of a homogeneous background $\left(I_{s h}=0.7, \theta=1.34\right)$. Solid lines represent stable solutions and dashed lines unstable ones.

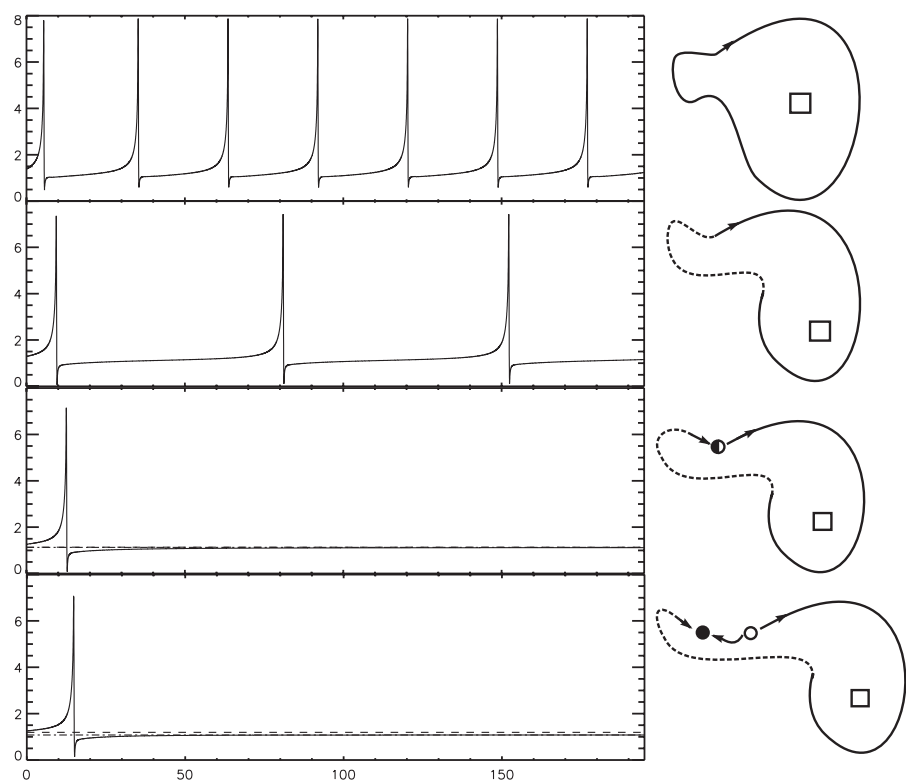

Figure 6: Left: Ls maximum intensity as a function of time for decreasing values of $I_{s h}$. From top to bottom, $I_{s h}=0.545,0.475,0.4471,0.435 . I_{s}=0.8050, \theta=1.34$. Right: sketch of the phase space for each parameter value.

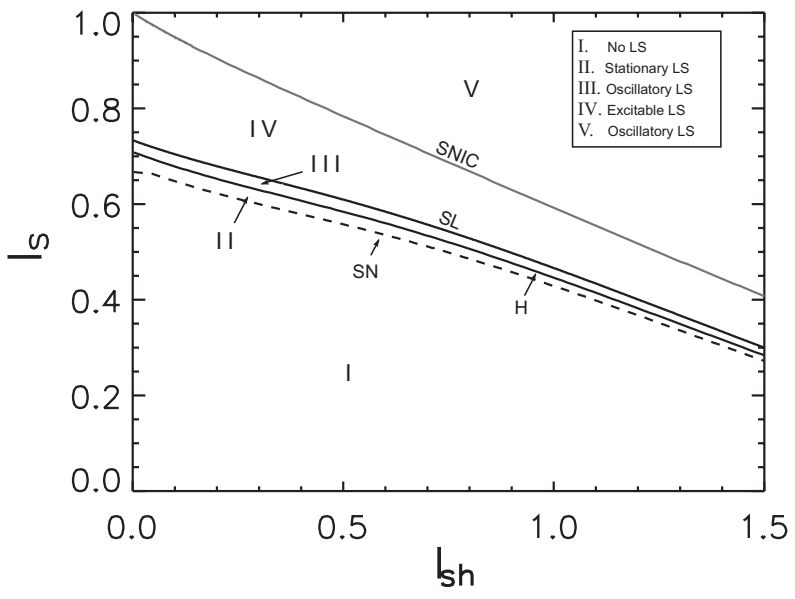

Figure 7: Phase diagram $I_{s}$ vs. $I_{s h}$ for $\theta=1.34$. 
furcation is encountered. This bifurcation, which is in fact a saddle-node on the invariant circle (SNIC) bifurcation is not present in the case of a homogeneous pump (cf. Fig. 1) for which the lower branch is stable until $I_{s}=1$. At the SNIC a middle branch unstable LS starts. As for homogeneous pump, there is a turning point (associated to the left saddle-node) where an stable upper-branch LS starts. Increasing $I_{s}$ the upper-branch LS undergoes a Hopf bifurcation resulting in a periodically oscillating localized structure. As for homogeneous pump this oscillatory structure is destroyed in a saddle-loop bifurcation leading to excitable behavior. The saddle-loop takes place at a value of $I_{s}$ below the SNIC. The excitable regime is possible only while the fundamental solution exists ( $I_{s}$ between the saddle-loop and the SNIC). After the SNIC both the fundamental and the upper branch LS are unstable, and a new, oscillatory, regime appears. For $I_{s}>1$ the background becomes modulationally unstable leading to pattern formation. Fig. 6 shows the temporal evolution in the new oscillatory regime. As $I_{s}$ is decreased the period of the oscillations becomes longer and it finally diverges when reaching the SNIC. The scaling of the period of oscillation confirms a saddle-node on the invariant circle bifurcation. A SNIC bifurcation induces excitable behavior for $I_{s}$ below the critical value. This scenario is different from the previous one although the excitable regimes are similar. Here the excitable threshold can be controlled by the intensity of the addressing Gaussian beam that effectively approaches the fixed point and the saddle in the phase space.

Figure 7 shows the phase diagram as a function of $I_{s}$ and $I_{s h}$ with $\theta$ constant. $I_{s h}=0$ corresponds to homogeneous pump. For small $I_{s}$ the system has only one fixed point which is the fundamental solution (reg. I). Increasing $I_{s}$ the occurrence of a saddle-node bifurcation leads to a stable and an unstable (saddle) branches of stationary LS solutions (reg. II). Further on, the stable branch becomes unstable in a Andronov-Hopf bifurcation and a cycle (oscillating LS) is created (reg. III). At this point a stable fixed point, a cycle and an unstable fixed point coexist in the system. If we further increase $I_{s}$ the limit cycle approaches the saddle and collides in a saddle-loop bifurcation (SL line). Beyond this saddle-loop the fundamental solution becomes excitable in two possible ways (reg. IV). If the line indicated as SNIC is crossed, the fundamental solution (stable) and the lower LS stationary solution (saddle) annihilate inside an invariant circle, leading to oscillatory LS behavior (reg. V). Region IV is excitable in the sense that suitable perturbations to the fundamental solution lead to long excursions in phase space, in two possible ways, depending on whether the system is close to the SL or to the SNIC bifurcation lines.

\section{ACKNOWLEDGMENT}

We thank G. Orriols and D. Pazó for useful discussions. We acknowledge financial support from MEC (Spain) and FEDER: Grants BFM2001-0341-C02-02, FIS2004-00953 (CONOCE2) and FIS2004-05073-C04-03. AJ acknowledges financial support from the MEC.

\section{REFERENCES}

1. Feature section on cavity solitons, L. A. Lugiato ed., IEEE J. Quant. Elect., Vol. 39, \#2, 2003; Tlidi, M., P. Mandel, and R. Lefever, Phys. Rev. Lett., Vol. 73, 640, 1994; Schapers, B., et al., ibid., Vol. 85, 748, 2000.

2. Barland, S., et al., Nature, Vol. 419, 699, 2002.

3. Firth, W. J. and A. J. Scroggie, Phys. Rev. Lett., Vol. 76, 1623, 1996; Firth, W. J. and C. O. Weiss, Opt. Photon. News, Vol. 13, 55, 2002.

4. Gomila, D., M. A. Matias, and P. Colet, Phys. Rev. Lett., Vol. 94, 063905, 2005.

5. Gomila, D., M. A. Matias, A. Jacobo, and P. Colet, "Phase space structure of 2D excitable localized structures," submitted for publication.

6. Meron, E., Phys. Rep., Vol. 218, 1, 1992; Murray, J. D., Mathematical Biology, 3rd Ed., Springer, 2002.

7. Plaza, F., et al., Europhys. Lett., Vol. 38, 85, 1997; Dubbeldam, J. L. A., B. Krauskopf, and D. Lenstra, Phys. Rev. E, Vol. 60, 6580, 1999; Wunsche, H. J., et al., Phys. Rev. Lett., Vol. 88, 023901, 2002; Barland, S., et al., Phys. Rev. E, Vol. 68, 036209, 2003.

8. Lugiato, L. A. and R. Lefever, Phys. Rev. Lett., Vol. 58, 2209, 1987.

9. Firth, W. J., et al., J. Opt. Soc. Am. B, Vol. 19, 747, 2002.

10. Skryabin, D., J. Opt. Soc. Am. B, Vol. 19, 529, 2002.

11. Guckenheimer, J. and P. Holmes, Nonlinear Oscillations, Dynamical Systems, and Bifurcations of Vector Fields, Springer, New York, 1983. 